\title{
James Richard Houck
}

Daniel Weedman and Donald BarryThomas Soifer

Citation: Physics Today 69, 1, 62 (2016); doi: 10.1063/PT.3.3059

View online: http://dx.doi.org/10.1063/PT.3.3059

View Table of Contents: http://physicstoday.scitation.org/toc/pto/69/1

Published by the American Institute of Physics

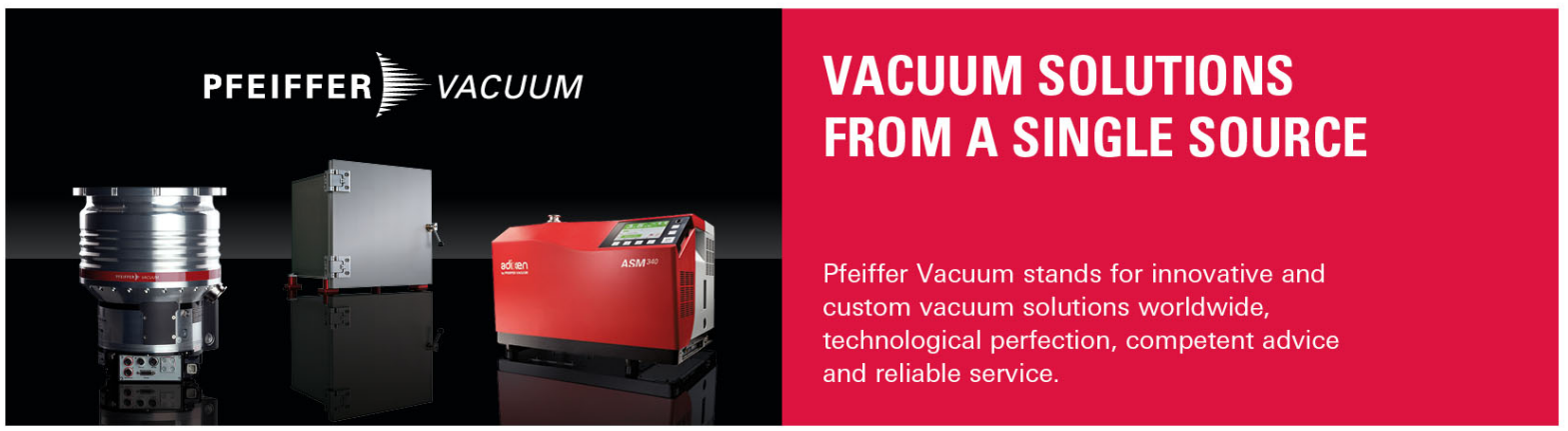


To notify the community about a colleague's death, subscribers can visit www.physicstoday.org/obituaries, where they can submit obituaries (up to 750 words), comments, and reminiscences. Each month recently posted material will be summarized here, in print. Select online obituaries will later appear in print.

\section{James Richard Houck}

ames Richard Houck, the leading figure in developing IR spectroscopy for astrophysics, died in Ithaca, New York, on 18 September 2015 from complications of Alzheimer's disease.

Born on 5 October 1940 in Mobile, Alabama, Jim received a BS in physics in 1962 from the Carnegie Institute of Technology. He spent his scientific career at Cornell University, starting in 1962 as a physics graduate student and remaining until his retirement as the Kenneth A. Wallace Professor of Astronomy in 2012. His passion for learning, doing, and teaching science by building instruments and understanding physics wrought great benefits for his students and astronomy colleagues.

After receiving his $\mathrm{PhD}$ in condensedmatter physics, he changed his field to astronomy, in which he collaborated initially with his colleague Martin Harwit. Jim emphasized building spectrographs and using ground-based, airborne, and rocket-borne IR instrumentation to make observations. He flew on every airplane NASA provided for astronomy, and he made numerous treks to the White Sands Missile Range in New Mexico to launch payloads on Aerobee sounding rockets.

Jim's observations with rockets and airplanes were primarily of galactic objects, including planetary nebulae, H II regions, and stars. The most notable airborne result was the discovery of water in the surface of Mars based on IR absorption at $2.85 \mu \mathrm{m}$. Jim was the lead author on a 1973 paper showing that "bound water comprises about one percent by weight of the surface material." The authors' analysis was confirmed 40 years later when the Mars rover Curiosity uncovered water content by vaporizing Martian soil.

An expert on detectors, Jim was a vital part of NASA's first major IR space mission, the Infrared Astronomical Satellite (IRAS, 1983), and solved some major problems. With little time remaining before launch, he came up with a new design that saved the short-circuited, 25$\mu \mathrm{m}$ detector array for the international mission. He made the first sketch of the revised wiring diagram on a napkin at a

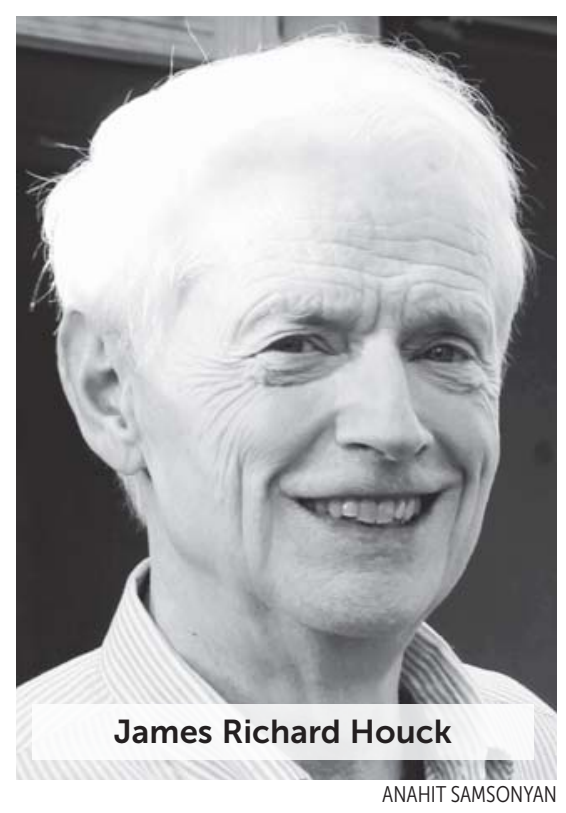

British pub. As the results came in, Jim became interested in extragalactic astronomy. He was a major participant in two of the most significant discoveries from IRAS, announced in 1984: a large population of optically faint, IR-bright galaxies and a new population of galaxies with exceptionally large intrinsic IR luminosities.

After the great success of IRAS, NASA began planning the Space Infrared Telescope Facility (SIRTF), now named the Spitzer Space Telescope. Managed by the Jet Propulsion Laboratory in Pasadena, California, Spitzer was successful primarily because of Jim and a very few other individuals. He was the principal investigator for SIRTF's IR spectrograph (IRS) and represented the IR viewpoint on the review panel, chaired by John Bahcall, that set astronomy's priorities for the 1990s (see Bahcall's article about the report, PHYSICS TODAY, April 1991, page 24). His advocacy convinced the panel that SIRTF should be the decade's highest priority. Subsequently, Jim chaired the NASA astrophysics advisory committee and was pivotal to organizing and participating in legislative advocacy for SIRTF. His extraordinary technical expertise was vital to SIRTF's essential redesign, which was needed to meet NASA cost targets.

Following the example of IRAS, SIRTF was originally intended primarily as a photometric mission. Jim advocated for a spectrograph, which became the instrument in greatest demand for the cryogenic Spitzer. His IRS improved sensitivity by orders of magnitude compared with previous IR spectroscopy. In 2005 in his first scientific paper containing IRS results, Jim and his colleagues announced the presence of a large population of early-universe quasars so obscured by dust that they had been invisible to optical telescopes.

The IRS's legacy is now permanently in place as CASSIS, the Cornell Atlas of Spitzer IRS Sources, which provides an easily accessible archive of mid-IR spectra (5-37 $\mu \mathrm{m}$ ) for everything from outerplanet satellites to quasars at the edge of the observable universe. CASSIS will be the fundamental reference archive of midIR spectra for the upcoming James Webb Space Telescope. For his contributions to IR astronomy, Jim received the NASA Exceptional Scientific Achievement Medal in 2005 and the American Astronomical Society's 2008 Joseph Weber Award for Astronomical Instrumentation.

Jim was dedicated to high-quality undergraduate teaching and to advising PhD students. His most notable legacy for undergraduates at Cornell was his development by 1974 of a research-quality telescope near campus. The 25-inch telescope of the Hartung-Boothroyd Observatory was used not only to train Cornell students but also to test spectrographs. One of Jim's undergraduate students related a sentiment felt by many: "I would not be anything close to what I am today had I not run into Jim in my first Cornell Astro course."

A few years before the 2003 SIRTF launch, his wife, Elaine, became partially disabled, and Jim devoted much time to her long-term care. He had assembled an extraordinary team to ensure the IRS's success, and he frequently hurried home from grueling trips to Pasadena to care for Elaine, who died in 2011. That combination of dedicated personal affection and incredible talent as an astrophysicist summarizes Jim's uniqueness.

$$
\begin{array}{r}
\text { Daniel Weedman } \\
\text { Donald Barry } \\
\text { Cornell University } \\
\text { Ithaca, New York } \\
\text { Thomas Soifer } \\
\text { California Institute of Technology } \\
\text { Pasadena PT }
\end{array}
$$

